\title{
MARgarete BAIER
}

\section{Sicherheit und Kontrolle im pflanzlichen Kraftwerk - Beiträge zur Regulation des plastidären antioxidativen Schutzsystems}

Pflanzen sind bis heute die wichtigsten Energieressourcen auf der Erde. In ihren grünen Teilen wandeln sie Lichtenergie in chemische Energie um. Diese kann von der Pflanze selbst oder von anderen Organismen sofort genutzt werden oder, wie im Falle von Erdöl und Kohle, über Jahrmillionen chemisch gebunden bleiben.

Die Energiewandelreaktion findet in der photosynthetischen Membran in den Chloroplasten statt. Chloroplasten sind semiautonome Organelle, die während der Evolution der Pflanzen durch die Aufnahme (Endosymbiose) von blaualgenähnlichen Zellen in nichtphotosynthetisch aktive eukaryotische Zellen entstanden sind. In den rezenten Pflanzen sind sie funktionell eng mit den sie umgebenden Zellstrukturen verbunden und tauschen mit ihnen Stoffwechselprodukte und Informationen aus. Während der evolutiven Reifung sind aus photosynthetisierenden prokaryotischen und heterotrophen eukaryotischen Vorläuferorganismen photosynthetisierende eukaryotische Lebewesen entstanden. Trotz aller Interaktion stellen Chloroplasten in den heutigen Pflanzen noch eigene Reaktionsräume dar und haben ihre genetische Eigenständigkeit nur teilweise aufgegeben. Während ca. 3.000 Genprodukte nach der Transkription im Zellkern und der Translation im Cytosol als Proteine in die Plastiden importiert werden, werden im Genom der Plastiden, dem Plastom, ca. 100 Proteine noch lokal kodiert. ${ }^{1}$ Die Überlebensfähigkeit der Pflanzen hängt aufgrund dieser Mischung von Genprodukten aus unterschiedlichen Kompartimenten entscheidend von der Effektivität und der Regulationsfähigkeit des Zusammenspiels von Plastiden und dem Zellkern ab. Uns interessieren dabei vor allem die Kommunikationsprozesse, die die plastidären antioxidativen Schutzmechanismen regulieren und die Effektivierung der Photosynthese während der Evolution der Pflanzen möglich gemacht haben und in rezenten Pflanzen die Umweltanpassungsfähigkeit prägen.

\section{Die Photosynthese als Triebkraft des Stoffwechsels}

Bei der Photosynthese werden durch Licht in den Chloroplasten Pigmente in den PigmentProteinkomplexen der Thylakoide, den Lichtsammelfallenkomplexen, angeregt. Im Idealfall geben sie die Anregungsenergie auf die Photoreaktionszentren weiter. Dort führt die Anregung zu einer Ladungstrennung. Dabei gibt das angeregte Chlorophyllmolekül ein Elektron an einen nachgeschalteten Elektronenakzeptor ab. Die entstandene Elektronenlücke wird durch die Reaktion mit Elektronendonatoren ausgeglichen. Durch Hintereinanderschalten von zwei Photosystemen und wiederholte Ladungstrennung kommt es im Licht in der Thylakoidmembran zum Elektronentransport. Durch Umsetzung der Licht-

1 Vgl. Leister und Schneider (2003). 
energie wird dabei die für biologische Systeme extreme Potenzialdifferenz von 1,55 V 2 überwunden, die sukzessiv als Triebkraft für Redoxreaktionen genutzt werden kann.

In der linearen Elektronentransportkette werden die Elektronen aus der photosynthetischen Elektronentransportkette auf das Reduktionsäquivalent $\mathrm{NADP}^{+}$oder das Thioredoxinsystem übertragen und dem Stoffwechsel für Reduktionsreaktionen zugeführt. Ein Großteil der Reduktionskraft wird im Calvin-Zyklus zur Reduktion von Kohlendioxid $\left(\mathrm{CO}_{2}\right)$ zu Kohlenhydraten genutzt. Parallel dazu fließen größere Mengen Reduktionsäquivalente in die Reduktion von Sulfat und Nitrat und andere Stoffwechselprozesse, so dass energievermittelnde und -speichernde Biomoleküle wie Kohlenhydrate, Aminosäuren und Fette synthetisiert werden können.

\section{Das zerstörerische Potenzial der photosynthetischen Lichtreaktion}

Die für biologische Systeme außergewöhnlich hohe Spannungsdifferenz von 1,55 V, die in der photosynthetischen Lichtreaktion aufgebaut wird, und die hohe Elektronegativität der angeregten Photoreaktionszentren und Redoxcarrier birgt allerdings auch ein großes Risiko für unkontrollierte Elektronentransferreaktionen. So können verstärkt Energie oder Elektronen von aktivierten Photoreaktionszentren und ihren elektronegativen Reaktionsprodukten auf Sauerstoff übertragen werden, wenn die Lichtintensität die Elektronentransportkapazität der photosynthetischen Elektronentransportkette übersteigt. Eine besondere Gefahr geht von den Photoreaktionszentren aus, die mit einem Redoxpotenzial von -900 $\mathrm{mV}$ zu den vermutlich elektronegativsten biologischen Reaktanten gehören. ${ }^{3}$

Durch Reduktion von Sauerstoff entstehen an der photosynthetischen Membran Superoxidanionen $\left(\mathrm{O}_{2}^{-}\right)$(Abb. 1). ${ }^{4}$ Daneben kann Sauerstoff bei Energieüberschuss durch direkten Energietransfer aus den Pigmenten $\mathrm{zu} \mathrm{O}_{2} *$ (Singulettsauerstoff) aktiviert werden. In wässriger Lösung können diese primären reaktiven Sauerstoffspezies (reactive oxygen species - ROS) zu Hydroxylradikalen $(\mathrm{OH})$ und Wasserstoffperoxid $\left(\mathrm{H}_{2} \mathrm{O}_{2}\right)$ weiterreagieren. Diese ebenfalls noch hochreaktiven Verbindungen greifen die unterschiedlichsten Biomoleküle an und beeinflussen die Struktur und Funktion der Zelle. ${ }^{5}$ Besonders anfällig sind ungesättigte Verbindungen, wie viele Fettsäuren der Biomembranen und aromatische Aminosäuren in Enzymen und Strukturproteinen. ${ }^{6}$ Diese können epoxidiert, peroxidiert und schließlich in Folge der Reaktion mit reaktiven Sauerstoffspezies gespalten werden. Unter Einfluss der chemischen Veränderungen werden die für die Kompartimentierung der Zelle so wichtigen Membranen durchlässig und Proteine denaturieren. ${ }^{7}$ Im Extremfall kann die unkontrollierte Freisetzung von reaktiven Sauerstoffspezies so zum Tod der Zellen, des Gewebes und schließlich der Pflanze führen.

In der Natur tritt die photooxidative Aktivierung von Sauerstoff stetig, zumindest in geringem Umfang, auf. Unter den meisten Bedingungen werden die schädlichen Produkte unmittelbar durch enzymatische und niedermolekulare Antioxidantien gequencht. Die Bildungsrate kann allerdings unter suboptimalen Lebensbedingungen stark ansteigen. Dies

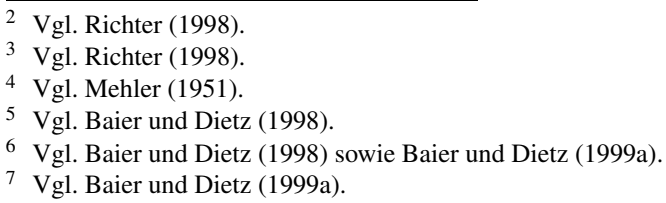




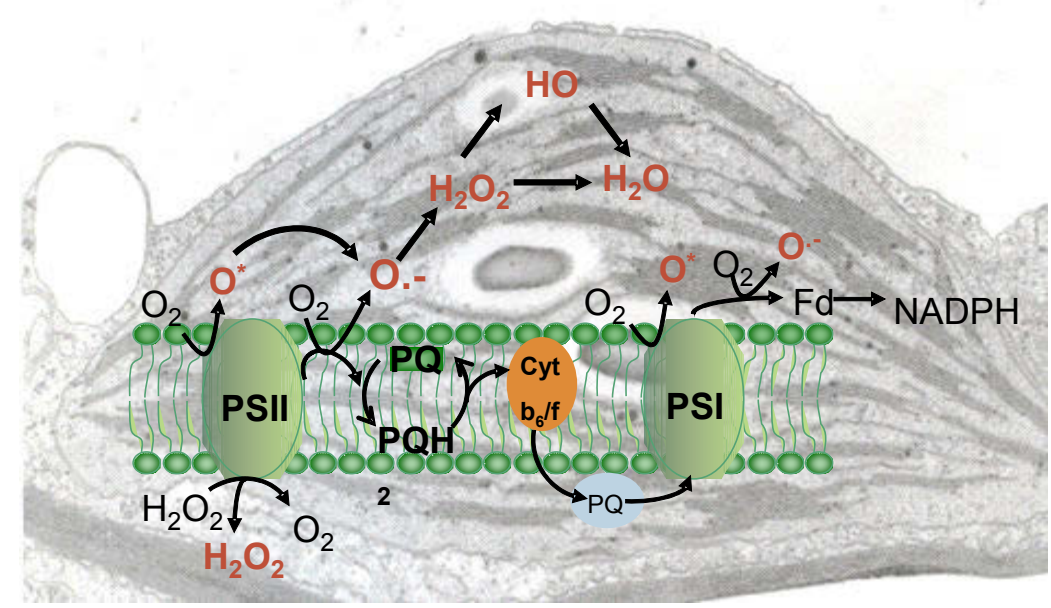

Abb. 1: Die Reaktionskette reaktiver Sauerstoffspezies. An den Photoreaktionszentren (PS-I und PSII) kann Sauerstoff zu Superoxid $\left(\mathrm{O}_{2}^{-}\right)$reduziert werden, das anschließend zu Wasserstoffperoxid $\left(\mathrm{H}_{2} \mathrm{O}_{2}\right)$ und Hydroxylradikalen $(\mathrm{HO})$ weiterreagieren kann.

ist insbesondere der Fall, wenn die Lichteinstrahlung höhere Elektronentransporteffizienzen ermöglichen kann, als der reduktive Stoffwechsel Elektronen aus den Elektronencarriersystemen, wie dem $\mathrm{NADP}^{+} / \mathrm{NADPH}$ und Thioredoxinpool, aufnehmen kann. Die daraus resultierenden Schäden sind in der Natur im Sommer an „Brandflecken“ und Blattvergilbungen (Chlorosen) erkennbar. Besonders kritisch sind Phasen, in denen Pflanzen nach mehreren schattigen Tagen plötzlich hohen Lichtintensitäten ausgesetzt werden. Die Gefährlichkeit der photosynthetischen Energiekonversionsreaktion kommt grundsätzlich vor allem vor dem Hintergrund der kurzzeitigen Schwankungen in den Umweltbedingungen zutage. Hinzu kommt, dass Verwundung, wie zum Beispiel infolge von Tierfraß oder Insektenbefall, und Pathogeninfektionen die metabolischen und energetischen Gleichgewichte verschieben können. Die Schäden treten dabei in der Regel akut auf, da sich Pflanzen, sofern die Umweltveränderungen nicht zu massiv sind, langfristig an die veränderten Situationen anpassen können.

\section{Das antioxidative Schutzsystem der Plastiden}

Da Licht als exogene Inputgröße für Pflanzen aufgrund ihrer sessilen Lebensweise nicht kontrollierbar ist, sind Pflanzen überhaupt nur lebens- und anpassungsfähig, weil sie während der Evolution ein Schutzsystem aus enzymatischen und nicht-enzymatischen Antioxidantien entwickelt haben. Dabei handelt es sich um ein kombinatorisches Netzwerk, in dem die einzelnen Komponenten mit unterschiedlicher Schutzkraft ineinandergreifen und sich teilweise in ihrer antioxidativen Wirkung ergänzen können. Im Falle des plastidären antioxidativen Schutzsystems ist es vermutlich durch Kombination von Genen und Metaboliten des Endosymbionten und des nicht-photosynthetisch aktiven, eukaryotischen Vorläuferorganismus entstanden, so dass man die Entstehung des plastidären antioxidativen 
Schutzsystems nur im Zusammenhang mit der Evolution der Pflanzen und dem Ausreifen der Endosymbiose zwischen Plastiden und heterotropher Vorläuferzelle verstehen kann.

Im antioxidativen Schutzsystem sind quantitativ besonders die enzymatischen Komponenten entscheidend: Im Halliwell-Asada-Foyer-Zyklus ${ }^{8}$ werden reaktive Sauerstoffspezies über das Zusammenspiel von Superoxid-Dismutasen (SOD) und Ascorbatperoxidasen (APx) unter Einsatz von Ascorbat zu Wasser entgiftet. Im ersten Schritt werden an der Thylakoidmembran durch die Superoxiddismutasen jeweils zwei Superoxidanionen in Sauerstoff und Wasserstoffperoxid $\left(\mathrm{H}_{2} \mathrm{O}_{2}\right)$ umgesetzt. $\mathrm{H}_{2} \mathrm{O}_{2}$ wiederum wird sukzessiv über die Ascorbatperoxidasen entgiftet, von denen zumindest bei den höheren Pflanzen meist eine stromale und eine thylakoidgebundene Form vorkommen. Als Nebenprodukte der ascorbatabhängigen Peroxidentgiftung entstehen Monodehydroascorbatradikale und Dehydroascorbat (Abb. 2). Das lebenswichtige niedermolekulare Antioxidans Ascorbat (Vitamin C) wird anschließend aus Monodehydroascorbat und Dehydroascorbat unter Verwendung des Thiols Glutathion über ein Enzymsystem aus Monodehydro- und Dehydroascorbatreduktasen (MDHAR; DHAR) und der Glutathionreduktase (GR) regeneriert.

In dieser Sekundärreaktion (Halliwell-Foyer-Zyklus) werden bei der Reduktion des Glutathions über die Glutathionreduktase Reduktionsäquivalente (NADPH) konsumiert. Durch die dabei ablaufende Regeneration von $\mathrm{NADP}^{+}$, das der bedeutendste Hauptelektronenakzeptor der photosynthetischen Lichtreaktion ist, vermindert das Schutzsystem neben der Entgiftung reaktiver Sauerstoffspezies den Elektronendruck in der photosynthetischen Membran.

Aufgrund der Empfindlichkeit der Ascorbatperoxidase gegen reaktive Sauerstoffspezies $^{9}$ ist der Halliwell-Asada-Zyklus jedoch in seiner Kapazität und Umweltreaktionsfähigkeit begrenzt. Weiterhin ist die Wirksamkeit des Halliwell-Asada-Zyklus in einigen Organismengruppen, wie verschiedenen Algen und Moosen, durch das Fehlen von Komponenten wie den $\mathrm{CuZn-Superoxiddismutasen} \mathrm{und} \mathrm{Monodehydroascorbatperoxidasen} \mathrm{ge-}$ schwächt. Puffernd wirken die niedermolekularen Antioxidantien, unter denen Ascorbat und Glutathion dominante Rollen einnehmen und auch in Abwesenheit der Enzyme reaktive Sauerstoffspezies inaktivieren können - wenn auch mit deutlich geringerer Rate.

Nach der Identifizierung des ersten pflanzlichen 2-Cys-Peroxiredoxins ${ }^{10}$ deutete sich vor gut zehn Jahren an, dass der Halliwell-Asada-Zyklus durch ein ascorbatunabhängiges, vermutlich innerhalb des Pflanzenreichs ubiquitäres alternatives Schutzsystem ergänzt wird, das wir heute den Peroxiredoxin-Zyklus (Abb. 3) ${ }^{11}$ nennen. Er wird, wie Folgeexperimente zeigten, durch vier parallel geschaltete Enzyme, zwei 2-Cys-Peroxiredoxine, ein Typ-II-Peroxiredoxin (PrxIIE) und ein atypisches Peroxiredoxin vom Q-Type (PrxQ), ${ }^{12}$ getrieben, die sich durch ihre Sequenz und ihre Expressionssteuerung unterscheiden. Anders als der Halliwell-Asada-Zyklus, der in höheren Pflanzen in der Schutzwirkung dominiert, deuten die in den vergangenen fünf Jahren möglich gewordenen Genomanalysen

\footnotetext{
8 Vgl. Asada (2000).

9 Vgl. Miyake und Asada (1996).

10 Vgl. Baier und Dietz (1996).

${ }^{11}$ Vgl. Dietz et al. (2003).

${ }^{12}$ Vgl. Horling et al. (2003) sowie Dietz et al. (2003).
} 


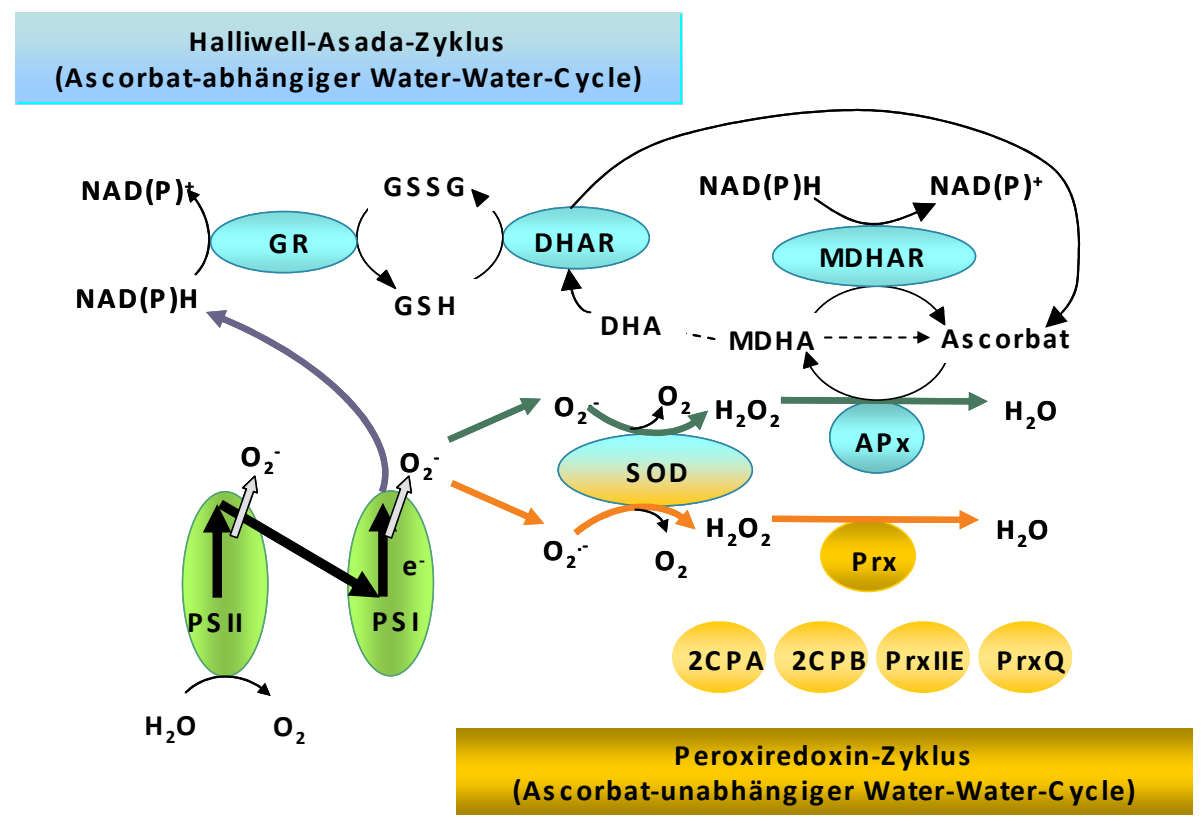

Abb. 2: ROS-Entgiftung: Über die Superoxid-Dismutase (SOD) und Ascorbatperoxidase (APx) werden im Halliwell-Asada-Zyklus (grün), beziehungsweise über Superoxid-Dismutasen und Peroxiredoxine (orange) im Peroxiredoxin-Zyklus, Superoxidanionen und Wasserstoffperoxid entgiftet. Die Regeneration des Ascorbats über die Monodehydroascorbat- und Dehydroascorbatreduktasen (MDHAR; DHAR) und Glutathionreduktasen (GR) werden Elektronen aus der photosynthetischen Lichtreaktion konsumiert (blau).

an, dass das Peroxiredoxinsystem evolutiv konserviert ist. Einzelne Pflanzengruppen unterscheiden sich nur in der Kopienzahl der einzelnen Peroxiredoxinuntergruppen. ${ }^{13}$

Vergleichende biochemische Analysen der Peroxiredoxine belegten, dass der Peroxiredoxin-Zyklus neben Wasserstoffperoxid $\left(\mathrm{H}_{2} \mathrm{O}_{2}\right)$ auch Alkylhydroperoxide ( $\left.\mathrm{ROOH}\right)$ entgiften kann, ${ }^{14}$ die durch Reaktion von reaktiven Sauerstoffspezies mit Biomolekülen entstehen. In der Peroxiredoxin-Reaktion reagieren die Peroxide, anders als bei den Ascorbatperoxidasen, in denen die Reaktion an selbst redoxempfindlichen Häm-Funktionen abläuft, direkt mit einem katalytischen Cysteinrest im Protein, wodurch sich die Peroxiredoxine als relativ stabile, aber auch verglichen mit den Ascorbatperoxidasen langsame Peroxidasen darstellen. Die Regeneration des in der Peroxidentgiftung zum Sulfensäurederivat (-SOH) oxidierten Cysteinrest erfolgt unter Beteiligung des zweiten proteinogenen Cysteinrests oder der Thiolgruppe eines niedermolekularen Thiols, wie dem Glutathion, ${ }^{15}$

\footnotetext{
${ }^{13}$ Vgl. Dietz et al. (2005).

${ }^{14}$ Vgl. Baier und Dietz (1997), König et al. (2002) sowie König et al. (2003).

${ }^{15}$ Vgl. Rouhier und Jacquot (2005).
} 


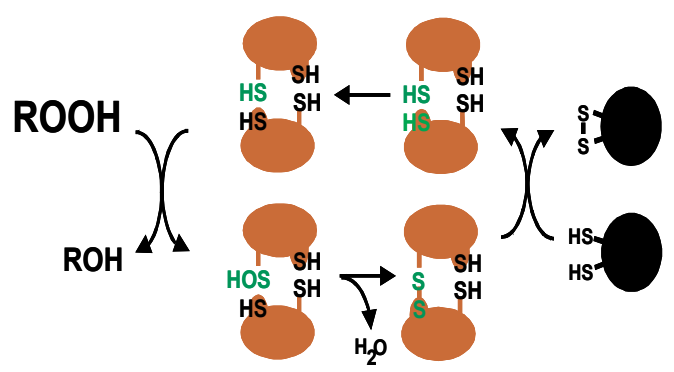

Abb. 3: Peroxidentgiftung am Beispiel des 2-Cys-Peroxiredoxins. Das Peroxiredoxin (Prx) reduziert $\mathrm{H}_{2} \mathrm{O}_{2}$ und Alkylhydroperoxide über einen intermolekularen Thiol-Disufid-Mechanismus (grün). Kleine Redoxproteine, wie die Thioredoxine (Trx), regenerieren das Enzym und konsumieren Elektronen am Photosystem I (PS-I).

und kleinen Redoxproteinen, wie den Thioredoxinen ${ }^{16}$ oder Glutaredoxinen ${ }^{17}$ (Abb. 3). Da die plastidären Redoxproteine über ferredoxinabhängige Enzyme an den photosynthetischen Elektronentransport funktional angekoppelt sind, ${ }^{18} \mathrm{kann}$ der peroxiredoxinvermittelte Entgiftungsmechanismus, wie der Halliwell-Asada-Foyer-Zyklus, ebenfalls gleichzeitig den Elektronendruck in der photosynthetischen Elektronentransportkette reduzieren und reaktive Sauerstoffspezies entgiften. ${ }^{19}$

Die durch Sequenzanalyse und biochemische Untersuchungen hergeleitete biologische Bedeutung im Schutz vor reaktiven Sauerstoffspezies wurde für das 2-Cys-Peroxiredoxin mittels Pflanzenlinien mit künstlich erniedrigtem Peroxiredoxinspiegel belegt. ${ }^{20}$ Die Testlinien zeigten entsprechend ihrer Limitierung im plastidären antioxidativen Schutzsystem für die Akkumulation reaktiver Sauerstoffspezies typische Schädigungen, wie Chlorosen (Abbau des grünen Blattfarbstoffs Chlorophyll) und Proteinabbau. ${ }^{21}$ In jungen Pflanzen war in Folge der Schädigung des Photosyntheseapparats die Photosyntheserate erniedrigt. Im weiteren Verlauf wurde in den 2-Cys-Peroxiredoxin verarmten Pflanzen die Expression und Aktivität des Halliwell-Asada-Zykluses als Komplementärsystem induziert, ${ }^{22}$ wodurch die Aktivität der photosynthetischen Lichtreaktion wieder gesteigert wurde. Hierdurch konnte gezeigt werden, dass die im Kern lokalisierten Gene gezielt in Abhängigkeit vom Schutzniveau in den Chloroplasten reguliert werden.

Da die Enzyme in allen Pflanzen von den einzelligen Algen, über Moose und Farne bis hin zu höheren Pflanzen konserviert sind, ${ }^{23}$ gehen wir davon aus, dass der Peroxiredoxin-Zyklus ein universeller Schutzmechanismus ist, der die Zellen vor schädlichen Übersprungsreaktionen wie der Energie- und Elektronenübertragung von der photosynthetischen Elektronentransportkette auf Sauerstoff schützt.

\footnotetext{
${ }^{16}$ König et al. (2002).

17 Vgl. Rouhier et al. (2002).

${ }^{18}$ Vgl. Buchanan und Balmer (2005).

${ }^{19}$ Vgl. Dietz et al. (2006).

${ }^{20}$ Vgl. Baier und Dietz (1999b) sowie Baier et al. (2000).

${ }^{21}$ Vgl. Baier und Dietz (1999b).

22 Vgl. Baier et al. (2000).

${ }^{23}$ Vgl. u.a. Horling et al. (2001) sowie Dietz et al. (2006).
} 


\section{Regulation der Schutzkapazität über Genexpressionssteuerung}

Sequenz- und Genomanalysen lassen vermuten, dass in allen grünen Pflanzen (Chlorobionten) alle Enzyme des chloroplastidären, antioxidativen Schutzsystems im Zellkern kodiert werden und folglich als Proteine posttranslational in die Chloroplasten eingeschleust werden müssen. ${ }^{24}$ Dies wirft die Frage auf, wie die Gene für chloroplastidäre antioxidative Enzyme im Zellkern so gesteuert werden können, dass die für die jeweilige Umweltsituation notwendigen Mengen an Genprodukten bereitgestellt werden können.

Bereits die Genexpressionsanalyse in 2-Cys-Peroxiredoxin verarmten Arabidopsispflanzen zeigte, dass die Genaktivität im Zellkern auf aus den Chloroplasten kommende Redoxsignale reagiert. ${ }^{25}$ Bis heute kennt man jedoch weder die chemische Natur der Signale, noch weiß man, wie sie übertragen werden. ${ }^{26}$ Potenzielle Signale können reaktive Sauerstoffspezies, aber auch redoxabhängig geprägte Metabolitsignaturen und spezifische Botenstoffe sein. ${ }^{27} \mathrm{Da}$ in der roten Pflanzenlinie, den Rhodobionten, einzelne Enzyme noch in den Plastiden kodiert sind, gehen wir davon aus, dass sich die Kommunikationswege zwischen Plastiden und Zellkern erst während der Evolution der grünen Pflanzen verfeinert hat.

Die Erkenntnislage über die Kommunikationswege zwischen Plastiden und Zellkern ist derzeit noch sehr gering. ${ }^{28}$ Während sich erste Vorstellungen zur Regulation der Gene für die Lichtsammelfallenkomplexe der photosynthetischen Membran während dem Ergrünen über Mg-Protoporphyrine ${ }^{29}$ und den abscisinsäureabhängigen Transkriptionsfaktor $\mathrm{ABI} 2^{30}$ abzeichnen, ist die Redoxkommunikation nahezu unerforscht. Neben reaktiven Sauerstoffspezies ${ }^{31}$ und kinaseabhängigen Signalen, die durch den Redoxzustand des Plastochinonpools in der photosynthetischen Lichtreaktion gesteuert werden, ${ }^{32}$ werden vor allem metabolische Signale postuliert, die durch die Akzeptorverfügbarkeit am Photosystem I kontrolliert werden. ${ }^{33}$ Letztere werden heute als bedeutendste Regulatoren eingestuft. ${ }^{34}$

\section{Identifizierung erster Regulatoren zur Steuerung der Gene für plastidäre antioxidative Enzyme}

Für die Regulation des 2-Cys-Peroxiredoxingens, dessen Transkriptionsaktivität mit der Akzeptorverfügbarkeit am Photosystem I korreliert, ${ }^{35}$ konnten wir inzwischen ein erstes Steuerelement im Promotor identifizieren, das auf Änderungen im Elektronendruck der photosynthetischen Lichtreaktion anspricht. ${ }^{36}$ Es aktiviert die Genexpression, wenn

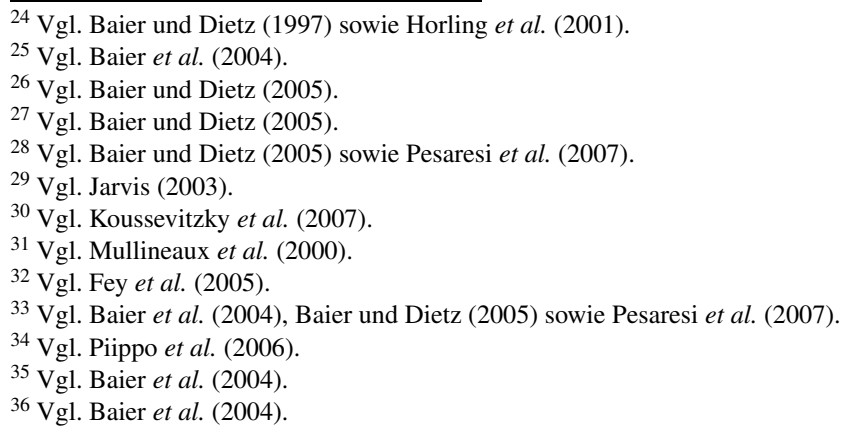


es in der photosynthetischen Elektronentransportkette zu einem Elektronenstau und damit zu einem erhöhten Risiko zur Bildung reaktiver Sauerstoffspezies kommt. Mit Hilfe dieses Steuerelements wurde in den vergangenen Jahren in Hefe unter 20.000 heterolog exprimierten Arabidopsis-Protein(fragment)en der Transkriptionsfaktor Rap2.4a als Regulator identifiziert. Der Expressionsaktivator bindet spezifisch an den Promotorabschnitt und reagiert auf Veränderungen in der zellulären Redoxsignatur, indem er nach leichten Redoxverschiebungen zunächst dimerisiert und schließlich unter extremen Stressbedingungen oligomerisiert. ${ }^{37}$ Die intermediäre, dimere Form stimuliert die Transkriptionsaktivität des 2-Cys-Peroxiredoxingens. In-vitro-Analysen des Transkriptionfaktors deuten an, dass diese Aktivierung zwischen der Überlastung des Glutathion- und Thioredoxinsystems einsetzt; also genau dann, wenn die Kapazität der vorhandenen Schutzsysteme überlastet ist und über die Genexpression im Zellkern das Schutzdefizit in den Plastiden ausgeglichen werden muss.

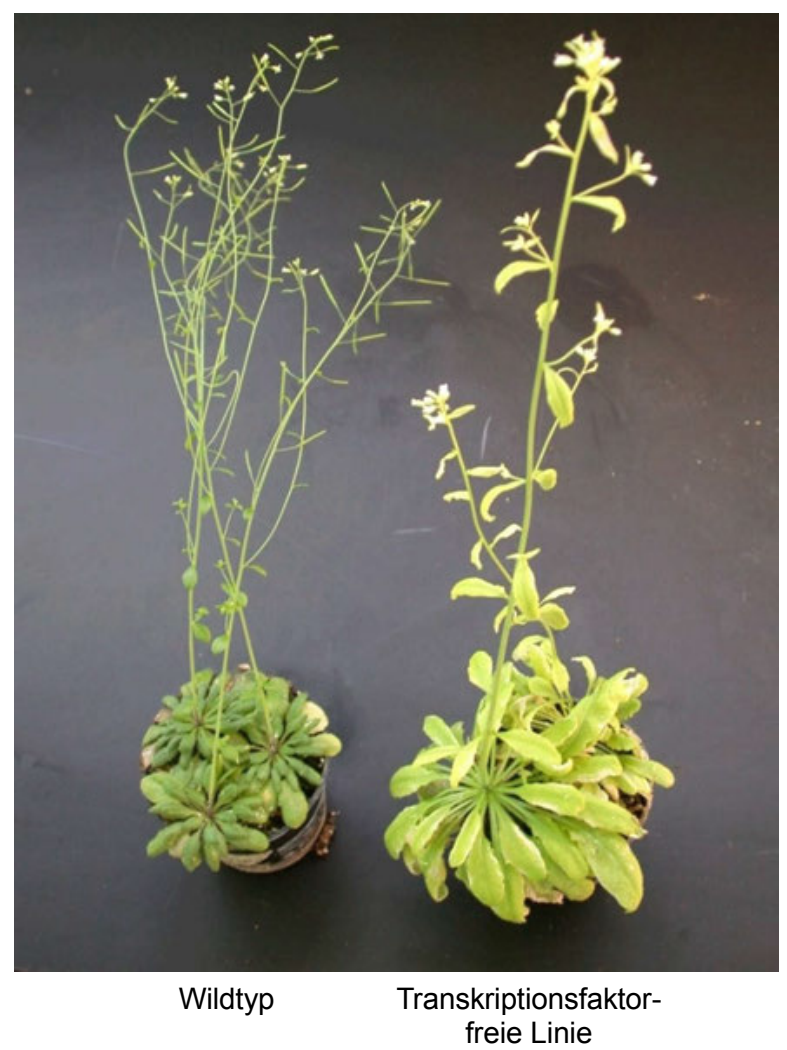

Abb. 4: Das Fehlen des regulierenden Transkriptionfaktors Rap2.4a führt bei Arabidopsis thaliana zu verringerter Umweltstabilität und die Pflanzen werden unter natürlich variierenden Umweltbedingungen chlorotisch.

${ }^{37}$ Vgl. Shaikhali et al. (2008). 


\section{Spezifität der redoxabhängigen Plastiden-Zellkern-Kommunikation}

Die vergleichende Analyse von Arabidopsispflanzen, die besagten Transkriptionsfaktor nicht mehr bilden können (Abb. 4), und Mutanten, die anderweitig in der Steuerung der 2-Cys-Peroxiredoxinexpression gestört sind, ${ }^{38}$ zeigen, dass sich die Regulation des plastidären Schutzsystems von der des extraplastidären Abwehrsystems abgrenzt. Das plastidäre Schutzsystem wird bereits bei leichten Verschiebungen der Redoxlage aktiviert. Bei starken Stressbedingungen wird es inaktiviert, wodurch der Photosyntheseapparat bei extremer Belastung ausgeschaltet werden kann. Der zelluläre Redoxschutz lastet dann auf den extraplastidären Kompartimenten, deren Schutzenzyme bei starken Redoxverschiebungen aktiviert werden. Anders als die Expression der plastidären antioxidativen Schutzenzyme, die im moderaten Stressbereich ein Maximum zeigt, steigt die Transkriptionsaktivität der bisher näher untersuchten Gene für cytosolische antioxidative Enyzme graduell mit der Verschiebung der zellulären Redoxlage, vermutlich durch ROS-Signale gesteuert, an und erreicht ihr Maximum kurz vor dem Absterben der Zellen. ${ }^{39}$

Aus den Unterschieden zwischen der Reaktion der Gene für plastidäre und cytosolische antioxidative Enzyme und der Reaktionsfähigkeit der kernlokalisierten Gene auf plastidäre Signale leiten wir ab, dass während der Evolution der Pflanzen ein spezifisches Kommunikationssystem zwischen den endosymbiotisch erworbenen Plastiden und dem Zellkern der ursprünglich heterotrophen eukaryotischen Zelle entstanden ist. Die Analyse der Funktionsweise dieses Kommunikationssystems und seiner regulativen Wirkung in der Umwelttoleranz und Adaptationsfähigkeit von Pflanzen in natürlichen und künstlichen Habitaten wird unsere Arbeit an der Heinrich-Heine-Universität prägen.

\section{Danksagung}

An dieser Stelle möchte ich mich bei allen bedanken, die diese Arbeit bisher mitgetragen haben und bei allen, die es uns möglich gemacht haben, die begonnene Arbeit in Düsseldorf fortzuführen und um neue Aspekte zu bereichern.

\section{Literatur}

AsADA, K. (2000). „The water-water cycle as alternative photon and electron sinks“, Philosophical Transactions of the Royal Society 355, 1419-1431.

BAIER, M. und K.-J. Dietz (1996). „Primary structure and expression of plant homologues of animal and fungal thioredoxin-dependent peroxide reductases and bacterial alkyl hydroperoxide reductases“, Plant Molecular Biology 31, 553-564.

BAIER, M. und K-J DIETZ (1997). „The plant 2-cys peroxiredoxin BAS1 is a nuclear encoded chloroplast protein: its expressional regulation, phylogenetic origin, and implications for its specific physiological function in plants“, The Plant Journal 12, 179-190.

BAIER, M. und K-J DIETZ (1998). ,The costs and benefits of oxygen for photosynthesizing plant cells“, Progress in Botany 60, 282-314.

BAIER, M. und K.-J. DiETZ (1999a).,,Alkyl hydroperoxides: the way out of the oxidative breakdown of lipids in chloroplasts“, Trends in Plant Science 4, 166-168.

\footnotetext{
${ }^{38}$ Vgl. Heiber et al. (2007).

${ }^{39}$ Vgl. Gadjev et al. (2006) sowie Mittler et al. (1999).
} 
BAIER, M. und K.-J. DIETZ (1999b). „Protective function of chloroplast 2-cysteine peroxiredoxin in photosynthesis. Evidence from transgenic Arabidopsis“, Plant Physiology 119, 1407-1414.

BAier, M., G. Noctor, C.H. Foyer und K.-J. Dietz (2000). „Antisense suppression of 2-cysteine peroxiredoxin in Arabidopsis specifically enhances the activities and expression of enzymes associated with ascorbate metabolism but not glutathione metabolism“, Plant Physiology 124, 823-832.

BAIER, M., E. StröHeR und K.-J. Dietz (2004). „The acceptor availability at photosystem I and ABA control nuclear expression of 2-Cys peroxiredoxin-A in Arabidopsis thaliana", Plant and Cell Physiology 45, 997-1006.

BAIER, M. und K.-J. DiETZ (2005). „Chloroplasts as source and target of cellular redox regulation: a discussion on chloroplast redox signals in the context of plant physiology", Journal of Experimental Botany 56, 1449-1462.

Buchanan, B.B. und Y. Balmer (2005). „Redox regulation: A broadening horizon“, Annual Reviews in Plant Biology 56, 187-220.

Dietz, K.-J., J. König, I. FinKeMeIER, P. LAMKEMEYER und M. BAieR (2003). „The function of plant peroxiredoxins: compartment-specific detoxification of peroxides with broad substrate specificity or redox sensors?", Free Radical Research 37, 9.

Dietz, K.J., T. Stork, I. Finkemeier, P. Lamkemeyer, WX Li, M. El-Tayeb, K.P. MiCHEL, E. Pistorius und M. BAIER (2005). „The role of peroxiredoxins in oxygenic photosynthesis of cyanobacteria and higher plants: peroxide detoxification or redox sensing?“, in: B. Demmig-Adams et al. (Hrsg.). Photoprotection, photoinhibition, gene regulation and environment. Dordrecht und New York, 303-319.

Dietz, K.-J., S. JACob, M.L. Oelze, M.V. LaXa, S.M.N. De Miranda, M. BAier und I. FinKeMEIER (2006). „The function of peroxiredoxins in plant organelle redox metabolism“, Journal of Experimental Botany 57, 1697-1709.

Fey, V., R. Wagner, K. Bräutigam, M. Wirtz, R. Hell, A. Dietzmann, D. Leister, R. OELMÜLlER und T. PFANNSCHMIDT (2005). ,,Retrograde plastid signals in the expression of nuclear genes for chloroplast proteins of Arabidopsis thaliana“, Journal of Biological Chemistry 280, 5318-5328.

Gadjev, I., S. Vanderauwera, T.S. Gechev, C. Laloi, I.N. Minkov, V. Shulaev, K. Apel, D. Inze, R. Mittler und F. VAN Breusegem (2006). „Transcriptomic footprints disclose specificity of reactive oxygen species in Arabidopsis“, Plant Physiology 141, 436-445.

Heiber, I., E. Ströher, B. RaAtz, I. Busse, U. Kahmann, M.W. Bevan, K.-J. Dietz und M. BAIER (2007). ,The rimb (redox imbalanced)-mutants of Arabidopsis thaliana differentiate signalling pathways for redox-regulation of chloroplast antioxidant enzymes“, Plant Physiology $143,1774-1788$.

Horling, F., M. BAIER und K.-J. Dietz (2001). „Redox-regulation of the expression of the peroxide-detoxifying chloroplast 2-Cys peroxiredoxin in the liver work Riccia fluitans", Planta 214, 283-287.

Horling, F., P. LAmkemeyer, J. König, I. Finkemeier, A. KAndlbinder, M. BAier und K.J. DIETZ (2003). „Divergent light-, ascorbate-, and oxidative stress-dependent regulation of expression of the peroxiredoxin gene family in Arabidopsis“, Plant Physiology 131, 317-325.

JARVIS, P. (2003). „Intercellular signalling: the language of the chloroplast“", Current Biology 13, R314-R316.

König, J., M. Baier, F. Horling, U. Kahmann, G. Harris, P. SchÜrmann und K.-J. Dietz (2002). ,The plant-specific function of 2-Cys peroxiredoxin-mediated detoxification of peroxides in the redox-hierarchy of photosynthetic electron flux “, Proceedings of the National Academy of Science, USA 99, 5738-5743. 
König, J., K. Lotte, R. Plessow, A. Brockhinke, M. Baier und K.-J. Dietz (2003). „Reaction mechanism of plant 2-Cys peroxiredoxin: Role of $\mathrm{t}$ he $\mathrm{C}$-terminus and the quaternary structure", Journal of Biological Chemistry 278, 24409-24420.

Koussevitzky, S., A. Nott, T.C. Mockler, F. Hong, G. Sachetto-Martins, M. Surpin, J. LiM, R. MitTLER und J. CHORY (2007). „Signals from chloroplasts converge to regulate nuclear gene expression“, Science 316, 715-719.

LEISTER, D. und A. SCHNEIDER (2003). „From genes to photosynthesis in Arabidopsis thaliana“, International Review of Cytology 228, 31-83.

MEHLER, A.H. (1951). „Studies on reactions of illuminated chloroplasts I. Mechanism of the reduction of oxygen and other Hill reagents“, Archives Biochemstry and Biophysics 33, 65-77.

Mittler, R., E. LAM, V. ShulaeV und M. Cohen (1999). „Signals controlling the expression of cytosolic ascorbate peroxidise during pathogen-induced programmed cell death in tobacco", Plant Molecular Biology 39, 1025-1035.

MiYAKE, C. und K. As ADA (1996). „Inactivation mechanism of ascorbate peroxidise at low concentrations of ascorbate: Hydrogen peroxide decomposes compound I of ascorbate peroxidise“, Plant Cell Physiology 37, 423-430.

Mullineaux, P., L. Ball, C. Escobar, B. Karpinska, G. Creissen und S. Karpinski (2000). ,Are diverse signalling pathways integrated in the regulation of Arabidopsis antioxidant defence gene expression in response to excess excitation energy?“, Philosophical Transactions of the Royal Society 355, 1531-1540.

Pesaresi, P., A. Schneider, T. Kleine und D. Leister (2007). „Interorganellar communication“, Current Opinions in Plant Biology 10, 600-606.

Piippo, M., Y. Allahverdiyeva, V. PaAkKarinen, U.M. Souranta, N. Battchikova und E.M. ARO (2006). „Chloroplast-mediated regulation of nuclear genes in Arabidopsis thaliana in the absence of light stress“, Physiological Genomics 25, 142-152.

RICHTER, G. (1998). Stoffwechselphysiologie der Pflanzen. Stuttgart und New York.

Rouhier, N., E. GelhaYe, J.P. JACQUOT (2002). „Glutaredoxin-dependent peroxiredoxin from poplar: protein-protein interaction and catalytic mechanism“, Journal of Biological Chemistry 277, 13609-13614.

RouHIER, N. und J.P. JACQUOT (2005). „The plant mulitgenic family of thiol peroxidases“, Free Radicals in Biology and Medicine 38, 1413-1421.

Shaikhali, J., I. Heiber, T. Seidel, E. Ströher, H. Hiltscher, S. Birkmann, K.-J. Dietz und M. BAIER (2008). ,,The redox-sensitive transcription factor Rap2.4a controls nuclear expression of 2-Cys peroxiredoxin A and other chloroplast antioxidant enzymes", BMC Plant Biology 8,48 . 
